\title{
LOCALLY LIPSCHITZ COMPOSITION OPERATORS AND APPLICATIONS TO NONLINEAR INTEGRAL EQUATIONS
}

\author{
J. APPELL, N. GUANDA AND YU. LYSAKOVA \\ Communicated by Paul Martin
}

\begin{abstract}
It is well known that imposing a global Lipschitz condition on nonlinear composition operators leads to a strong degeneracy phenomenon in many function spaces. In contrast to this, we show that a local version of Banach's contraction mapping principle is less restrictive and applies to a large variety of nonlinear problems. We illustrate this by means of applications to nonlinear integral equations with bounded or weakly singular kernels.
\end{abstract}

1. Nonlinear composition operators. Given a function $h$ : $[0,1] \times \mathbf{R} \rightarrow \mathbf{R}$ and a space $X$ of functions $f:[0,1] \rightarrow \mathbf{R}$, we call as usual the operator $H: X \rightarrow X$ defined by

$$
H f(t)=h(t, f(t)) \quad(0 \leq t \leq 1)
$$

the composition operator (or Nemytskij operator or superposition operator) generated by $h$. In the particular case when the function $h$ does not depend on the first variable, i.e., $h: \mathbf{R} \rightarrow \mathbf{R}$, the corresponding operator

$$
H f(t)=(h \circ f)(t)=h(f(t)) \quad(0 \leq t \leq 1)
$$

2010 AMS Mathematics subject classification. 47H30, Secondary 26A16, 26A45, 45G05, 45G10.

Keywords and phrases. Composition operator, global Lipschitz condition, local Lipschitz condition, functions of bounded variation, Hölder continuous functions, contraction mapping principle, nonlinear integral equation.

This paper was written while the third author was a DAAD fellow (Grant \# A/10/86156) in Würzburg, Germany. Financial support of the DAAD and hospitality of the University of Würzburg are gratefully acknowledged. The second author is indebted to CDCH (Venezuela) for financial support (Grant \# 03-82362011/1.)

Received by the editors on May 13, 2012, and in revised form on November 7, 2012.

DOI: $10.1216 /$ JIE-2013-25-3-321

Copyright (C)2013 Rocky Mountain Mathematics Consortium 
is known as the autonomous composition operator. The operators (1.1) and (1.2) will be studied on the closed ball

$$
B_{r}(X)=\left\{f \in X:\|f\|_{X} \leq r\right\} \quad(r>0)
$$

in several function spaces $X$, with a particular emphasis on two spaces of functions of bounded variation. The first one is the space $W B V_{p}[0,1]$ with norm

$$
\|f\|_{W B V_{p}}=|f(0)|+\operatorname{WVar}_{p}(f ;[0,1])^{1 / p},
$$

where

$$
\operatorname{WVar}_{p}(f ;[0,1]):=\sup \left\{\sum_{j=1}^{m}\left|f\left(t_{j}\right)-f\left(t_{j-1}\right)\right|^{p}\right\}
$$

denotes the total $p$-variation (in Wiener's sense [33]) of $f$ on $[0,1]$, and the supremum in (1.5) is taken over all partitions $\left\{t_{0}, t_{1}, \ldots, t_{m}\right\}$ of $[0,1]$. The second one is the space $R B V_{p}[0,1]$ with norm

$$
\|f\|_{R B V_{p}}=|f(0)|+\operatorname{RVar}_{p}(f ;[0,1])^{1 / p},
$$

where

$$
\operatorname{RVar}_{p}(f ;[0,1]):=\sup \left\{\sum_{j=1}^{m} \frac{\left|f\left(t_{j}\right)-f\left(t_{j-1}\right)\right|^{p}}{\left(t_{j}-t_{j-1}\right)^{p-1}}\right\}
$$

denotes the total $p$-variation (in Riesz's sense $[\mathbf{3 0}]$ ) of $f$ on $[0,1]$, and the supremum in (1.7) is again taken over all partitions $\left\{t_{0}, t_{1}, \ldots, t_{m}\right\}$ of $[0,1]$.

Obviously, for $p=1$, both spaces $W B V_{p}[0,1]$ and $R B V_{p}[0,1]$ reduce to the familiar space $B V[0,1]$ of functions of bounded variation. For $p>1$, however, these spaces have quite different properties. For instance, the scale of spaces $W B V_{p}[0,1]$ is increasing in $p$, while the scale of spaces $R B V_{p}[0,1]$ is decreasing in $p$, i.e.,

$W B V_{p}[0,1] \subset W B V_{p+\varepsilon}[0,1], \quad R B V_{p}[0,1] \supset R B V_{p+\varepsilon}[0,1] \quad(\varepsilon>0)$.

Moreover, both spaces $W B V_{p}[0,1]$ and $R B V_{p}[0,1]$ contain the space Lip $[0,1]$ of Lipschitz continuous functions $f:[0,1] \rightarrow \mathbf{R}$ with norm

$$
\|f\|_{\text {Lip }}=|f(0)|+\operatorname{lip}(f),
$$


where

$$
\operatorname{lip}(f):=\sup _{s \neq t} \frac{|f(s)-f(t)|}{|s-t|},
$$

and both spaces are continuously imbedded into the space of bounded functions with norm

$$
\|f\|_{\infty}=\sup _{0 \leq t \leq 1}|f(t)|
$$

Interestingly, the space $W B V_{p}[0,1]$ also contains, for $0<\alpha \leq 1 / p$, the space $\operatorname{Lip}_{\alpha}[0,1]$ of Hölder continuous functions $f:[0,1] \rightarrow \mathbf{R}$ with norm

$$
\|f\|_{\operatorname{Lip}_{\alpha}}=|f(0)|+\operatorname{lip}_{\alpha}(f),
$$

where

$$
\operatorname{lip}_{\alpha}(f):=\sup _{s \neq t} \frac{|f(s)-f(t)|}{|s-t|^{\alpha}},
$$

while the space $R B V_{p}[0,1]$ does not contain any Hölder space. In fact, in $[4$, Exercise 14.29] the authors construct a sophisticated function

$$
f \in\left(\bigcap_{0<\alpha<1} \operatorname{Lip}_{\alpha}[0,1]\right) \backslash B V[0,1]
$$

By the chain of inclusions (1.8), such a function cannot belong to $R B V_{p}[0,1](p \geq 1)$ either.

The most interesting property of the space $R B V_{p}[0,1]$, however, is given by the so-called Riesz lemma [30] which reads as follows: A function $f$ belongs to $R B V_{p}[0,1]$ for $p>1$ if and only if $f$ is absolutely continuous and its derivative $f^{\prime}$ (which exists almost everywhere) belongs to $L_{p}[0,1]$. A corresponding result is of course not true for $p=1$, since functions in $B V[0,1]$ need not be continuous, let alone absolutely continuous.

Let us now return to the composition operator $H$ defined in (1.1) and (1.2). A crucial problem related to this operator is to find conditions on the function $h$, possibly both necessary and sufficient, under which the corresponding operator $H$ maps a given function space $X$ into itself. Solving this problem is in some cases almost trivial, 
in some cases surprisingly difficult, and some cases simply unknown. In the autonomous case (1.2), one may give a complete answer for many important spaces; for further reference we summarize with the following.

Theorem 1.1. Let $h: \mathbf{R} \rightarrow \mathbf{R}$ be given, and let $X$ be any of the spaces $\operatorname{Lip}[0,1], \operatorname{Lip}_{\alpha}[0,1], A C[0,1], B V[0,1], W B V_{p}[0,1]$, or $R B V_{p}[0,1]$. Then the following are equivalent:

(a) The function $h$ satisfies the local Lipschitz condition:

$$
|h(u)-h(v)| \leq k(r)|u-v| \quad(|u|,|v| \leq r) .
$$

(b) The operator (1.2) generated by $h$ maps $X$ into itself.

Theorem 1.1 was proved for $X=\operatorname{Lip}$ and $X=\operatorname{Lip}_{\alpha}$ in $[\mathbf{3}, \mathbf{2 9}$, for $X=A C$ in [25], for $X=B V$ in [11], for $X=W B V_{p}$ in [1], and for $X=R B V_{p}$ in $[\mathbf{2 4}, \mathbf{2 7}]$. This result also holds in other spaces, like the space $H B V$ of all functions of bounded harmonic variation [7]. We point out that in $[\mathbf{1}]$ it is shown that the condition $H($ Lip $) \subseteq B V$ (which is weaker than the condition $H(X) \subseteq X$ for all spaces $X$ occurring in Theorem 1.1) implies (1.12); the converse is trivially true.

One may also prove that, under the hypothesis (1.12) on $h$, the operator $H$ is automatically bounded in the norm of all spaces $X$ occurring in Theorem 1.1. On the other hand, proving continuity of $H$ is much more difficult. (Recall that, in contrast to linear operators, a nonlinear operator may be bounded and discontinuous, or continuous and unbounded.) In [8] it is shown that the operator $H$ in (1.2) is continuous in the norm (1.9) of $X=\operatorname{Lip}$ or (1.11) of $\operatorname{Lip}_{\alpha}$ if and only if $h \in C^{1}(\mathbf{R})$. As far as we know, finding necessary and sufficient conditions on $h$ for the continuity of $H$ in one of the spaces $B V, A C$, $W B V_{p}$, or $R B V_{p}$ is an open problem.

2. Global and local Lipschitz conditions. A useful tool for solving nonlinear problems is to apply fixed point principles, the most commonly used being Schauder's and Banach's fixed point theorems. Clearly, for applying these theorems, just boundedness and continuity of the operators involved does not suffice, but one needs some strong additional properties. 
A certain flaw of Schauder's theorem is that it requires the knowledge of compactness criteria, possibly both necessary and sufficient, which in some function spaces (like spaces of Hölder continuous functions) are unknown, and in other function spaces (like spaces of functions of bounded variation) are known, but very clumsy and technical. It is therefore a useful device to try first to apply Banach's contraction mapping principle which is much simpler.

However, when doing so, one encounters another serious drawback. In fact, imposing a (global) Lipschitz condition like

$$
\|H f-H g\|_{X} \leq K\|f-g\|_{X} \quad(f, g \in X)
$$

to the composition operator (1.1) in some function space $X$, even with a very large Lipschitz constant $K$, often leads to a strong degeneracy for the generating function $h$ : the operator $H$ satisfies (2.1) if and only if the corresponding function $h$ has the form

$$
h(t, u)=\alpha(t)+\beta(t) u \quad(0 \leq t \leq 1)
$$

for two functions $\alpha, \beta \in X$ (resp. constants $\alpha, \beta \in \mathbf{R}$ in the autonomous case $(1.2))$, i.e., the functions $h(t, \cdot)$ is affine. Such a degeneracy phenomenon was discovered first by Matkowski [18] in the space Lip of Lipschitz continuous functions. Subsequently, this was proved in many other spaces, namely, in [19] for the space $C^{n}$ of $n$ times continuously differentiable functions, in [16] for the space $\operatorname{Lip}_{\alpha}$ of Hölder continuous functions, in $[\mathbf{1 2}]$ for the space $\operatorname{Lip}^{n}$ of functions whose $n$th derivative is Lipschitz continuous, in [13] for the space $\operatorname{Lip}_{\alpha}^{n}$ of functions whose $n$th derivative is Hölder continuous, in [31] for the space $A C^{n}$ of functions whose $n$th derivative is absolutely continuous, in $[\mathbf{2 3}, \mathbf{2 6}]$ for the space $W B V_{p}$ for $p>1$ (see $[\mathbf{2 7},(1.4)]$ ) for the space $R B V_{p}$ for $p>1$ (see $[\mathbf{2 1},(1.6)])$ for the Sobolev space $W_{p}^{1}$ for $p>1$, and in $[\mathbf{1 7}, \mathbf{2 0}]$ for other function spaces. In [1] (see also [28]) it is proved that the global Lipschitz condition

$$
\|H f-H g\|_{Y} \leq K\|f-g\|_{X} \quad(f, g \in X)
$$

for $H$ acting between different spaces $X$ and $Y$ always implies that the corresponding function $h$ is of the form (2.2), provided that $X$ contains all affine functions and $Y \hookrightarrow \operatorname{Lip}$, i.e., $Y$ is continuously imbedded into the space of all Lipschitz continuous functions with norm (1.9). 
A similar, but different degeneracy holds in the space $B V[0,1]$, where one has to replace the function $h$ with its "left regularization" $h^{\#}$ defined by

$$
h^{\#}(t, u)= \begin{cases}h(0, u) & \text { for } t=0 \\ \lim _{s \rightarrow t-} h(s, u) & \text { for } 0<t \leq 1 .\end{cases}
$$

Indeed, Matkowski and Miś proved in $[\mathbf{2 2}]$ that the operator (1.1) satisfies (2.1) in $X=B V[0,1]$ if and only if

$$
h^{\#}(t, u)=\alpha(t)+\beta(t) u \quad(0 \leq t \leq 1)
$$

with suitable functions $\alpha, \beta \in B V[0,1]$. More generally, in [1], it is proved that condition (2.3) for $H: X \rightarrow Y$ always implies that the regularization $h^{\#}$ of $h$ satisfies (2.4), provided that $X$ contains all polynomial functions and $Y \hookrightarrow W B V_{p}$ for some $p \geq 1$ (e.g., $Y=B V$ ).

The described degeneracy phenomenon means that, loosely speaking, we may apply Banach's fixed point principle in theses spaces only if the problem under consideration is actually linear, and therefore not very interesting. Closer scrutiny of many nonlinear problems reveals, however, that in most cases it suffices to impose a local Lipschitz condition of the form

$$
\|H f-H g\|_{X} \leq K(r)\|f-g\|_{X} \quad\left(\|f\|_{X},\|g\|_{X} \leq r\right),
$$

where the Lipschitz constant $K(r)$ in (2.5) essentially depends on the radius $r$ of the ball in which we impose this condition (and, as a matter of fact, satisfies $K(r) \rightarrow \infty$ as $r \rightarrow \infty$, see below). It turns out that (2.5) is much less restrictive than (2.1) and applies to a large variety of nonlinear problems. Moreover, in many spaces one may give, at least in the autonomous case (1.2), a necessary and sufficient condition in terms of the function $h$ under which the operator $H$ satisfies (2.5). We summarize with the following.

Theorem 2.1. Let $h: \mathbf{R} \rightarrow \mathbf{R}$ be given, and let $X$ be any of the spaces $\operatorname{Lip}[0,1], \operatorname{Lip}_{\alpha}[0,1], A C[0,1], B V[0,1], W B V_{p}[0,1]$, or $R B V_{p}[0,1]$. Then the following are equivalent:

(a) The derivative $h^{\prime}$ of $h$ satisfies the local Lipschitz condition

$$
\left|h^{\prime}(u)-h^{\prime}(v)\right| \leq k_{1}(r)|u-v| \quad(|u|,|v| \leq r) .
$$


(b) The operator (1.2) generated by $h$ satisfies (2.5).

Unfortunately, there is no "universal" proof of Theorem 2.1 for all these spaces, but every space requires a separate proof. The first result of this type was proved for $X=\operatorname{Lip}_{\alpha}$ in [32], but the proof given there is wrong. A complete proof for $\operatorname{Lip}_{\alpha}$ and all other spaces $X$ occurring in Theorem 2.1 may be found in [2].

It is illuminating to study the "interaction" between the minimal Lipschitz constant $k_{1}(r)$ for $h^{\prime}$ in (2.6) and the minimal Lipschitz constant $K(r)$ for $H$ in (2.5). Consider the characteristic

$$
\widetilde{k}_{1}(r):=\sup \left\{\left|h^{\prime}(u)\right|:|u| \leq r\right\}
$$

which is of course related to $k_{1}(r)$ from (2.6) through the simple estimate $\tilde{k}(r) \leq|h(0)|+r k(r)$. In [2] it is shown that $K(r)$ from (2.5) may be estimated by

$$
K(r) \leq \max \left\{r k_{1}(r), \widetilde{k}_{1}(r)\right\}
$$

if $k_{1}(r)$ from (2.6) is known, and, vice versa, $k_{1}(r)$ may be estimated by

$$
k_{1}(r) \leq \frac{2 K(2 r)+1}{r}
$$

if $K(r)$ is known. The last estimate has an interesting consequence. If (2.1) holds, i.e., $K$ can be chosen independent of $r$, then (2.9) implies that $k_{1}(r) \rightarrow 0$ as $r \rightarrow \infty$. So (2.6) implies that $h^{\prime}$ is actually constant which means that $h$ has the form (2.4) (with $\alpha, \beta \in \mathbf{R}$ ), recovering the above degeneracy result. This argument shows even more precisely that, for $h$ being non-affine, the function $K=K(r)$ in (2.5) must not only depend on $r$ but even satisfy

$$
\liminf _{r \rightarrow \infty} \frac{K(r)}{r}>0
$$

i.e., be of superlinear growth for large values of $r$.

3. Applications to regular integral equations. In this section we apply the abstract results given above to obtain existence (and 
sometimes uniqueness) of solutions of certain nonlinear integral equations. We start with an existence and uniqueness result for solutions of generalized bounded variation. To get a unified approach we use the shortcut $\operatorname{Var}_{p}(f)$ to denote either $\operatorname{WVar}_{p}(f ;[0,1])$ or $\operatorname{RVar}_{p}(f ;[0,1])$, and $B V_{p}$ to denote either the space $W B V_{p}[0,1]$ or the space $R B V_{p}[0,1]$.

Consider the nonlinear integral equations of Hammerstein type

$$
f(s)=\int_{0}^{1} k(s, t) h(t, f(t)) d t+b(s) \quad(0 \leq s \leq 1) .
$$

Here $k:[0,1] \times[0,1] \rightarrow \mathbf{R}$ is a given kernel function (whose properties will be made precise below), and $b \in B V_{p}$ is also given. We are interested in conditions on the nonlinearity $h:[0,1] \times \mathbf{R} \rightarrow \mathbf{R}$ under which (3.1) admits a (unique) solution $f \in B V_{p}$.

Denoting the corresponding linear integral operator in (3.1) by

$$
K g(s):=\int_{0}^{1} k(s, t) g(t) d t \quad(0 \leq s \leq 1)
$$

we may rewrite (3.1) as the operator equation

$$
f-K H f=b,
$$

where $H$ is the composition operator (1.1), and try to apply the familiar fixed point principles to (3.2). To this end, we suppose that the operator (3.1) maps the space $B V_{p}$ into itself and is bounded. For further reference we put

$$
k(r):=\sup \left\{\frac{|h(t, u)-h(t, v)|}{|u-v|}: 0 \leq t \leq 1,|u|,|v| \leq r, u \neq v\right\}
$$

and

$$
\widetilde{k}(r):=\sup \{|h(t, u)|: 0 \leq t \leq 1,|u| \leq r\}
$$

Concerning the kernel function $k$ in (3.1), we impose the following hypotheses. Suppose that $k(s, \cdot) \in L_{1}[0,1]$ for $0 \leq s \leq 1$, and that the function $v_{p}:[0,1] \rightarrow \mathbf{R}$ defined by

$$
v_{p}(t):=\operatorname{Var}_{p}(k(\cdot, t))^{1 / p}
$$


belongs to $L_{p}[0,1]$. To simplify the notation, we use the shortcut $(3.5)$

$\kappa_{p}:=\int_{0}^{1}|k(0, t)| d t+\left(\int_{0}^{1} \operatorname{Var}_{p}(k(\cdot, t)) d t\right)^{1 / p}=\|k(0, \cdot)\|_{L_{1}}+\left\|v_{p}\right\|_{L_{p}}$.

In Lemma 3.1 we give a sufficient condition under which the operator $f \mapsto K H f+b$ (whose fixed points coincide with the solutions of (3.2)) leaves a closed ball in the space $W B V_{p}[0,1]$ invariant. In case $p=1$, i.e., in the space $B V[0,1]$, similar conditions of this type have been considered in $[\mathbf{5}, \mathbf{6}]$.

Lemma 3.1. Under the above hypotheses, let $r>0$ be so large that

$$
\kappa_{p} \widetilde{k}(r)<r
$$

where $\widetilde{k}(r)$ is given by (3.4). Then the operator $f \mapsto K H f+b$ maps, for any $b \in B V_{p}$ satisfying

$$
\|b\|_{B V_{p}} \leq r-\kappa_{p} \widetilde{k}(r),
$$

the closed ball $B_{r}\left(B V_{p}\right)$ into itself.

Proof. For the sake of definiteness, let us prove the assertion in the space $W B V_{p}[0,1]$. Suppose that $r$ satisfies (3.6) and $b$ satisfies (3.7). Given $f \in B_{r}\left(W B V_{p}\right)$ and a partition $\left\{s_{0}, s_{1}, \ldots, s_{m}\right\}$ of $[0,1]$, we get

$$
\begin{aligned}
\sum_{j=1}^{m} \mid K H f\left(s_{j}\right) & -\left.\operatorname{KHf}\left(s_{j-1}\right)\right|^{p} \\
& =\sum_{j=1}^{m}\left|\int_{0}^{1}\left[k\left(s_{j}, t\right)-k\left(s_{j-1}, t\right)\right] h(t, f(t)) d t\right|^{p} \\
& \leq \sup _{0 \leq t \leq 1}|h(t, f(t))|^{p} \int_{0}^{1} \sum_{j=1}^{m}\left|k\left(s_{j}, t\right)-k\left(s_{j-1}, t\right)\right|^{p} d t \\
& \leq \widetilde{k}(r)^{p} \int_{0}^{1} v_{p}(t)^{p} d t,
\end{aligned}
$$

and so, passing to the supremum over all partitions $\left\{s_{0}, s_{1}, \ldots, s_{m}\right\}$,

$$
\operatorname{WVar}_{p}(K H f ;[0,1]) \leq \widetilde{k}(r)^{p}\left\|v_{p}\right\|_{L_{p}}^{p} .
$$


Consequently, from (3.7) we conclude that

$$
\begin{aligned}
\|K H f+b\|_{W B V_{p} \leq} & |K H f(0)|+\operatorname{WVar}_{p}(K H f ;[0,1])^{1 / p} \\
& +\|b\|_{W B V_{p}} \\
\leq & \int_{0}^{1}|k(0, t) h(t, f(t))| d t \\
& +\widetilde{k}(r)\left(\int_{0}^{1} v_{p}(t)^{p} d t\right)^{1 / p}+\|b\|_{W B V_{p}} \\
\leq & \widetilde{k}(r)\|k(0, \cdot)\|_{L_{1}}+\widetilde{k}(r)\left\|v_{p}\right\|_{L_{p}}+\|b\|_{W B V_{p}} \\
\leq & \widetilde{k}(r) \kappa_{p}+\|b\|_{W B V_{p}} \leq r,
\end{aligned}
$$

which proves the assertion for $W B V_{p}$. The proof for $R B V_{p}$ is exactly the same, with obvious modifications in the definition of variations.

In Lemma 3.2 we give a sufficient condition under which the operator $f \mapsto K H f+b$ is a contraction in norm (1.4), respectively (1.6).

Lemma 3.2. Under the above hypotheses, let $r>0$ be so small that

$$
\kappa_{p} k(r)<1,
$$

where $k(r)$ is given by (3.3). Then the operator $f \mapsto K H f+b$ is, for any $b \in B V_{p}[0,1]$, a contraction on $B_{r}\left(B V_{p}\right)$ with respect to the norm (1.4), respectively (1.6).

Proof. We work again in the space $W B V_{p}[0,1]$. Suppose that $r$ satisfies (3.8), and let $f, g \in B_{r}\left(W B V_{p}\right)$ be fixed. We claim that

$$
\|K H f-K H g\|_{W B V_{p}} \leq \kappa_{p} k(r)\|f-g\|_{W B V_{p}},
$$

which, together with (3.8), proves the assertion. First of all, we have

$$
\begin{aligned}
|K H f(0)-K H g(0)| & =\left|\int_{0}^{1} k(0, t)[h(t, f(t))-h(t, g(t))] d t\right| \\
& \leq k(r) \int_{0}^{1}|k(0, t)||f(t)-g(t)| d t \\
& \leq k(r)\|k(0, \cdot)\|_{L_{1}}\|f-g\|_{\infty} \\
& \leq k(r)\|k(0, \cdot)\|_{L_{1}}\|f-g\|_{W B V_{p}},
\end{aligned}
$$


where we have used the fact that $W B V_{p}[0,1]$ is continuously imbedded into the space of all bounded functions on $[0,1]$ with the supremum norm (1.10).

On the other hand, we now show that also

$$
\operatorname{WVar}_{p}(K H f-K H g ;[0,1])^{1 / p} \leq \kappa_{p} k(r)\|f-g\|_{W B V_{p}} .
$$

For any partition $\left\{s_{0}, s_{1}, \ldots, s_{m}\right\}$ of $[0,1]$, we have

$$
\begin{aligned}
\sum_{j=1}^{m} \mid K H f\left(s_{j}\right)- & K H f\left(s_{j-1}\right)-K H g\left(s_{j}\right)+\left.K H g\left(s_{j-1}\right)\right|^{p} \\
= & \sum_{j=1}^{m} \mid \int_{0}^{1} k\left(s_{j}, t\right)[H f(t)-H g(t)] d t \\
& -\left.\int_{0}^{1} k\left(s_{j-1}, t\right)[H f(t)-H g(t)] d t\right|^{p} \\
\leq & \|H f-H g\|_{\infty}^{p} \sum_{j=1}^{m} \int_{0}^{1}\left|k\left(s_{j}, t\right)-k\left(s_{j-1}, t\right)\right|^{p} d t \\
\leq & \|H f-H g\|_{W B V_{p}}^{p} \int_{0}^{1} \sum_{j=1}^{m}\left|k\left(s_{j}, t\right)-k\left(s_{j-1}, t\right)\right|^{p} d t \\
\leq & k(r)^{p}\|f-g\|_{W B V_{p}}^{p} \int_{0}^{1} v_{p}(t)^{p} d t \\
= & k(r)^{p}\left\|v_{p}\right\|_{L_{p}}^{p}\|f-g\|_{W B V_{p}}^{p} .
\end{aligned}
$$

Passing again to the supremum with respect to all partitions $\left\{s_{0}, s_{1}, \ldots\right.$, $\left.s_{m}\right\}$ of $[0,1]$, we obtain

$$
\operatorname{WVar}_{p}(K H f-K H g ;[0,1]) \leq k(r)^{p}\left\|v_{p}\right\|_{L_{p}}^{p}\|f-g\|_{W B V_{p}}^{p},
$$

and combining this with (3.9) yields

$$
\begin{aligned}
\|K H f-K H g\|_{W B V_{p}} \leq & k(r)\|k(0, \cdot)\|_{L_{1}}\|f-g\|_{W B V_{p}} \\
& +k(r)\left\|v_{p}\right\|_{L_{p}}\|f-g\|_{W B V_{p}} \\
= & k(r) \kappa_{p}\|f-g\|_{W B V_{p}},
\end{aligned}
$$

and establishes the result. 
Combining Lemmas 3.1 and 3.2, and observing that the fixed points of the operator $f \mapsto K H f+b$ coincide with the solutions of (3.2), Banach's classical contraction mapping theorem implies the following theorem.

Theorem 3.3. Suppose that there exists an $r>0$ such that both estimates (3.6) and (3.8) hold. Then the nonlinear integral equation (3.1) has, for each $b \in B V_{p}$ satisfying (3.7), a unique solution $f \in B V_{p}$.

Let us return for a moment to the autonomous case of operator (1.2) where, as one could expect, our calculations slightly simplify. For instance, the characteristic (3.4) takes here the simpler form

$$
\widetilde{k}(r)=\sup \{|h(u)|:|u| \leq r\} .
$$

We suppose in addition that the kernel function splits into two functions of one variable, i.e., $k(s, t)=l(s) m(t)$, where $l \in B V_{p}[0,1]$ and $m \in L_{p}[0,1]$. In this case, we get

$$
v_{p}(t)=\operatorname{Var}_{p}(l)^{1 / p}|m(t)| ;
$$

hence,

$$
\begin{aligned}
\kappa_{p} & =\int_{0}^{1}|l(0)||m(t)| d t+\left(\int_{0}^{1} \operatorname{Var}_{p}(l)|m(t)|^{p} d t\right)^{1 / p} \\
& =|l(0)|\|m\|_{L_{1}}+\operatorname{Var}_{p}(l)^{1 / p}\|m\|_{L_{p}} \leq\|l\|_{B V_{p}}\|m\|_{L_{1}} .
\end{aligned}
$$

Therefore, the crucial condition (3.6) in Lemma 3.1 holds if

$$
\|l\|_{B V_{p}}\|m\|_{L_{1}} \widetilde{k}(r)<r,
$$

with $\widetilde{k}(r)$ given by (3.10), while the crucial condition (3.8) in Lemma 3.3 holds if

$$
\|l\|_{B V_{p}}\|m\|_{L_{1}} k(r)<1
$$

with $k(r)$ given by (1.12). We illustrate these conditions by a very elementary example. 
Example 3.4. First let $h(u)=u^{\alpha}$, where $\alpha \in \mathbf{R}, \alpha \geq 2$. The mean value theorem shows that

$$
k(r)=\alpha r^{\alpha-1}, \quad \widetilde{k}(r)=r^{\alpha}, \quad k_{1}(r)=\alpha(\alpha-1) r^{\alpha-2}, \quad \widetilde{k}_{1}(r)=\alpha r^{\alpha-1}
$$

So estimate $(3.12)$ reads

$$
K(r) \leq\|l\|_{B V_{p}}\|m\|_{L_{1}} \alpha r^{\alpha-1}<1
$$

while the general estimate (2.8) from Section 2 becomes

$$
K(r) \leq\|l\|_{B V_{p}}\|m\|_{L_{1}} \max \left\{\alpha(\alpha-1) r^{\alpha-2}, \alpha r^{\alpha-1}\right\}<1 .
$$

Of course, both estimates may be achieved for $r$ sufficiently small, but (3.13) is better than (3.14) for $r<\alpha-1$, while (3.14) is better than (3.13) for $r>\alpha-1$.

Now let $h(u)=\log (1+u)$ for $u>0$ and $h(u)=0$ for $u \leq 0$. Again, the mean value theorem implies that

$$
k(r) \equiv 1, \quad \widetilde{k}(r)=\log (1+r), \quad k_{1}(r)=\frac{1}{(1+r)^{2}}, \quad \widetilde{k}_{1}(r)=\frac{1}{1+r} .
$$

So estimate $(3.12)$ reads

$$
K(r) \leq\|l\|_{B V_{p}}\|m\|_{L_{1}}<1
$$

while the estimate (2.8) becomes

$$
K(r) \leq\|l\|_{B V_{p}}\|m\|_{L_{1}} \max \left\{\frac{r}{(1+r)^{2}}, \frac{1}{1+r}\right\}=\frac{\|l\|_{B V_{p}}\|m\|_{L_{1}}}{1+r}<1 .
$$

Estimate (3.15) does not depend on $r$, but estimate (3.16) may be achieved for $r$ sufficiently small; so, in this example, (3.16) is always better than (3.15).

Let us point out that there is a sharp contrast between the autonomous operator (1.2) and the non-autonomous operator (1.1). Theorem 1.1 states that, for the operator (1.2), the local Lipschitz condition (1.12) is both necessary and sufficient for the inclusions $H\left(B V_{p}\right) \subseteq B V_{p}$. For proving this inclusion for the non-autonomous operator (1.1), it 
seems natural to impose a local Lipschitz condition on $h(t, \cdot)$ on $\mathbf{R}$, uniformly with respect to $t \in[0,1]$, together with the requirement $h(\cdot, u) \in B V_{p}[0,1]$, uniformly with respect to $u \in \mathbf{R}$. In fact, this combination of two conditions was stated in [14] for the space $B V$ without proof, and afterwards used as "obvious" by several authors. However, tempting as this idea appears at first glance, on reflection it becomes less convincing. Only quite recently it was shown by Maćkowiak [15] by means of a counterexample that this is in fact false! So even the harmless-looking problem of finding a sufficient condition for the inclusion $H(B V) \subseteq B V$ for operator (1.1) which is possibly not "too far" from being necessary, is open.

4. Applications to singular integral equations. In this section we apply our abstract results to a class of singular integral equations. Consider the nonlinear weakly singular Abel-Volterra equation

$$
f(s)-\int_{0}^{s} \frac{k(s, t) h(f(t))}{|s-t|^{\nu}} d t=b(s) \quad(0 \leq s \leq 1),
$$

where $k:[0,1] \times[0,1] \rightarrow \mathbf{R}$ is continuous and $0<\nu<1$. We rewrite (4.1) again as the operator equation

$$
f-K_{\nu} H f=b, \quad f \in X,
$$

where $K_{\nu}$ is the weakly singular linear integral operator defined by

$$
K_{\nu} g(s)=\int_{0}^{s} \frac{k(s, t) g(t)}{|s-t|^{\nu}} d t \quad(0 \leq s \leq 1),
$$

and $H$ is the autonomous composition operator (1.2). A very suitable space for treating such an operator equation is the Hölder space $\operatorname{Lip}_{\alpha}^{0}[0,1]$ of all functions $f \in \operatorname{Lip}_{\alpha}[0,1]$ satisfying $f(0)=0$. The socalled second Hardy-Littlewood theorem (see, e.g., $[\mathbf{9}]$ ) states that operator (4.3) maps $\operatorname{Lip}_{\alpha}^{0}[0,1] \cap L_{p}[0,1]$ into $\operatorname{Lip}_{\alpha}^{0}[0,1]$ and is bounded if

$$
\frac{1}{1-\nu}<p \leq \infty, \quad 0<\alpha \leq 1-\nu-\frac{1}{p} .
$$

In order to simplify our computations, let us choose $p=\infty$, so

$$
0<\alpha \leq 1-\nu, \quad \operatorname{Lip}_{\alpha}^{0}[0,1] \cap L_{\infty}[0,1]=\operatorname{Lip}_{\alpha}^{0}[0,1] .
$$


By Theorem 2.1, the local Lipschitz condition (1.12) is necessary and sufficient for operator (1.2) to satisfy (2.5) in Hölder spaces. Using Banach's fixed point principle in these spaces, we may deduce existence and uniqueness results for equation (4.1) in a closed ball of appropriate radius if $\|b\|_{\operatorname{Lip}_{\alpha}}$ is sufficiently small.

To explain this in more detail, we now prove two lemmas which are parallel to Lemmas 3.1 and 3.2. Throughout the remaining part of this section, we suppose for simplicity that $h(0)=0$ and denote by $\left\|K_{\nu}\right\|_{\alpha}$ the norm of operator (4.3) in the space $\operatorname{Lip}_{\alpha}$. Estimates or even explicit formulas for $\left\|K_{\nu}\right\|_{\alpha}$ in terms of the kernel $k$ in (4.1) may be found in $[\mathbf{1 0}]$.

Lemma 4.1. Under the hypotheses (1.12) and $h(0)=0$, the operator (1.2) maps the space $\operatorname{Lip}_{\alpha}^{0}[0,1]$ into itself and is bounded. Moreover, if $r>0$ is so large that

$$
\left\|K_{\nu}\right\|_{\alpha} k(r)<r
$$

where $k(r)$ is given by (1.12), then the operator $f \mapsto K_{\nu} H f+b$ maps, for any $b \in \operatorname{Lip}_{\alpha}[0,1]$ satisfying

$$
\|b\|_{\operatorname{Lip}_{\alpha}} \leq r-\left\|K_{\nu}\right\|_{\alpha} k(r)
$$

the closed ball $B_{r}\left(\operatorname{Lip}_{\alpha}\right)$ into itself.

Proof. The sufficiency of (1.12) for the boundedness of operator (1.2) in $\operatorname{Lip}_{\alpha}[0,1]$ follows from a trivial calculation. Moreover, it is easy to see that

$$
\sup \left\{\|H f\|_{\operatorname{Lip}_{\alpha}}:\|f\|_{\operatorname{Lip}_{\alpha}} \leq r\right\} \leq k(r)
$$

in this case, and so $H$ is bounded. Finally, our assumption $h(0)=0$ guarantees that operator (1.2) maps $\operatorname{Lip}_{\alpha}^{0}[0,1]$ into itself.

Fix $f \in \operatorname{Lip}_{\alpha}^{0}[0,1]$ with $\|f\|_{L_{i p_{\alpha}}} \leq r$, where $r$ satisfies (4.4), and suppose that $b \in \operatorname{Lip}_{\alpha}^{0}[0,1]$ satisfies (4.5). Then

$$
\begin{aligned}
\left\|K_{\nu} H f+b\right\| & \leq\left\|K_{\nu}\right\|_{\alpha} \sup _{\|f\|_{\operatorname{Lip} \alpha} \leq r}\|H f\|_{\operatorname{Lip}_{\alpha}}+\|b\|_{\operatorname{Lip}_{\alpha}} \\
& \leq\left\|K_{\nu}\right\|_{\alpha} k(r)+\|b\|_{\operatorname{Lip}_{\alpha}} \leq r
\end{aligned}
$$


by (4.5) and (4.6), which proves the assertion.

Lemma 4.2. Suppose that the derivative $h^{\prime}$ of $h: \mathbf{R} \rightarrow \mathbf{R}$ exists and satisfies (2.6), and let $\widetilde{k}_{1}(r)$ be defined by (2.7). Then, for $\left|\xi_{1}\right|,\left|\xi_{2}\right|,\left|\eta_{1}\right|,\left|\eta_{2}\right| \leq r$ we have

$$
\begin{aligned}
\mid h\left(\xi_{1}\right)-h\left(\eta_{1}\right)-h\left(\xi_{2}\right) & +h\left(\eta_{2}\right) \mid \\
\leq & \tilde{k}_{1}(r)\left|\xi_{1}-\eta_{1}-\xi_{2}+\eta_{2}\right| \\
& +k_{1}(r) \max \left\{\left(\left|\xi_{1}-\xi_{2}\right|+\left|\eta_{1}-\eta_{2}\right|\right)\left|\xi_{1}-\eta_{1}\right|,\right. \\
& \left.\left(\left|\xi_{1}-\eta_{1}\right|+\left|\xi_{2}-\eta_{2}\right|\right)\left|\xi_{1}-\xi_{2}\right|\right\} .
\end{aligned}
$$

The proof of the technical Lemma 4.2 consists in a repeated tricky application of the mean value theorem and may be found in $[\mathbf{2}]$. We apply the result to prove the following.

Lemma 4.3. Suppose that the derivative $h^{\prime}$ of $h$ exists and satisfies (2.6). Moreover, let $r>0$ be so small that

$$
k_{1}(r)<\frac{1}{\left\|K_{\nu}\right\|_{\alpha} 4 r}, \quad \widetilde{k}_{1}(r)<\frac{1}{\left\|K_{\nu}\right\|_{\alpha}},
$$

where $k_{1}(r)$ is given by (2.6) and $\widetilde{k}_{1}(r)$ by $(2.7)$. Then the operator $f \mapsto K H f+b$ is, for any $b \in \operatorname{Lip}_{\alpha}^{0}[0,1]$, a contraction on $B_{r}\left(\operatorname{Lip}_{\alpha}^{0}\right)$ with respect to the norm (1.11).

Proof. Fix $s, t \in[0,1]$ and $f, g \in \operatorname{Lip}_{\alpha}^{0}[0,1]$ with $\|f\|_{\operatorname{Lip}_{\alpha}} \leq r$ and $\|g\|_{\operatorname{Lip}_{\alpha}} \leq r$. Applying Lemma 4.2 to the choice $\xi_{1}:=f(s), \xi_{2}:=f(t)$, $\eta_{1}:=g(s)$ and $\eta_{2}:=g(t)$, yields

$$
\begin{aligned}
& |H f(s)-H g(s)-H f(t)+H g(t)| \\
\leq & \widetilde{k}_{1}(r)|f(s)-g(s)-f(t)+g(t)| \\
& +k_{1}(r)\|f-g\|_{\infty} \max \{(|f(s)-f(t)|+|g(s)-g(t)|), 2|f(s)-f(t)|\} .
\end{aligned}
$$

Dividing by $|s-t|^{\alpha}$, passing to the supremum over $s, t \in[0,1], s \neq t$, and observing that $\alpha<\beta$, we arrive at

$$
\begin{aligned}
\operatorname{lip}_{\alpha}(H f-H g) \leq \widetilde{k}_{1}(r) l i p_{\alpha}(f-g) & \\
& +k_{1}(r)\|f-g\|_{\infty} \max \left\{\operatorname{lip}_{\alpha}(f)+\operatorname{lip}_{\alpha}(g), 2 \operatorname{lip}_{\alpha}(f)\right\} .
\end{aligned}
$$


Since $\|f\|_{\operatorname{Lip}_{\alpha}}=\operatorname{lip}_{\alpha}(f)$ and $\|f\|_{\infty} \leq \operatorname{lip}(f)$ for $f \in \operatorname{Lip}_{\alpha}^{0}[0,1]$, (4.8) means nothing else but

$$
\left\|K_{\nu} H f-K_{\nu} H g\right\|_{\operatorname{Lip}_{\alpha}} \leq\left\|K_{\nu}\right\|_{\alpha} \operatorname{lip}_{\alpha}(H f-H g) \leq K(r)\|f-g\|_{\operatorname{Lip}_{\alpha}}
$$

where

$$
K(r):=\left\|K_{\nu}\right\|_{\alpha} \max \left\{4 r k_{1}(r), \widetilde{k}_{1}(r)\right\}<1,
$$

by assumption (4.7).

Combining Lemma 4.1 with Lemma 4.3, and observing that the fixed points of the operator $f \mapsto K H f+b$ coincide with the solutions of (4.1), Banach's classical contraction mapping theorem implies the following.

Theorem 4.4. Under the hypotheses of Lemmas 4.1 and 4.3, the nonlinear Abel-Volterra equation (4.1) has, for each sufficiently small $b \in \operatorname{Lip}_{\alpha}^{0}[0,1]$, a unique solution $f \in \operatorname{Lip}_{\alpha}^{0}[0,1]$.

Analyzing the proof of Theorem 4.4 shows that necessarily $k(r) \rightarrow \infty$ as $r \rightarrow \infty$ in (4.4), apart from the trivial case of an affine function $h$, in correspondence with the main result in [16]. Therefore, we cannot expect global existence and uniqueness of solutions of (4.1) in the whole space $\operatorname{Lip}_{\alpha}^{0}[0,1]$. This again justifies the necessity of replacing the global condition (2.1) by the local condition (2.5).

Acknowledgments. We are indebted to both referees for valuable comments. In particular, we are very grateful to the referee who pointed our attention to the forthcoming paper [15].

\section{REFERENCES}

1. J. Appell, N. Guanda, N. Merentes and J. L. Sánchez, Boundedness and continuity properties of nonlinear composition operators: A survey, Comm. Appl. Anal. 15 (2011), 153-182.

2. J. Appell, N. Guanda and M. Väth, Function spaces with the Matkowski property and degeneracy phenomena for nonlinear composition operators, Fixed Point Theory (Cluj) 12 (2011), 265-284.

3. A.A. Babaev, The structure of a certain nonlinear operator and its application, Azer. Gos. Univ. Uchen. Z. 4 (1961), 13-16 (in Russian). 
4. A.N. Bakhvalov, M.I. Dyachenko, K.S. Kazaryan, P. Sifuentes and P.L. Ul'yanov, Real analysis in exercises, Fizmatlit, Moscow, 2005 (in Russian).

5. D. Bugajewska, On the superposition operator in the space of functions of bounded variation, revisited, Math. Comp. Model. 52 (2010), 791-796.

6. - - On BV-solutions of some nonlinear integral equations, Int. Eq. Oper. Th. 46 (2003), 387-398.

7. M. Chaika and D. Waterman, On the invariance of certain classes of functions under composition, Proc. Amer. Math. Soc. 43 (1974), 345-348.

8. M. Goebel and F. Sachweh, On the autonomous Nemytskij operator in Hölder spaces, Z. Anal. Anw. 18 (1999), 205-229.

9. R. Gorenflo and S. Vessella, Abel integral equations, Lect. Notes Math. 1461, Springer, Berlin 1991.

10. A.I. Gusejnov and Kh.Sh. Mukhtarov, Introduction to the theory of nonlinear singular integral equations, Nauka, Moscow, 1980 (in Russian).

11. M. Josephy, Composing functions of bounded variation, Proc. Amer. Math. Soc. 83 (1981), 354-356.

12. J. Knop, On globally Lipschitzian Nemytskii operator in special Banach space of functions, Fasc. Math. 21 (1990), 79-85.

13. M. Lupa, Form of Lipschitzian operator of substitution in some class of functions, Z. Nauk. Pol. Łódz 21 (1989), 87-96.

14. A.G. Lyamin, On the acting problem for the Nemytskij operator in the space of functions of bounded variation, 11th School Theory Oper. Function Spaces, Chel'yabinsk (1986), 63-64 (in Russian).

15. P. Maćkowiak, A counterexample to Lyamin's theorem, Proc. Amer. Math. Soc., to appear.

16. A. Matkowska, On characterization of Lipschitzian operator of substitution in the class of Hölder's functions, Z. Nauk. Politech. Lódz. Mat. 17 (1984), 81-85.

17. A. Matkowska, J. Matkowski and N. Merentes, Remark on globally Lipschitzian composition operators, Demonstr. Math. 28 (1995), 171-175.

18. J. Matkowski, Functional equations and Nemytskij operators, Funkc. Ekv. Ser. Int. 25 (1982), 127-132.

19. - Form of Lipschitz operators of substitution in Banach spaces of differentiable functions, Z. Nauk. Politech. Łódz. Mat. 17 (1984), 5-10.

20. - Lipschitzian composition operators in some function spaces, Nonlin. Anal. TMA 30 (1997), 719-726.

21. J. Matkowski and N. Merentes, Characterization of globally Lipschitzian composition operators in the Sobolev space $W_{p}^{n}[a, b]$, Z. Nauk. Politech. Łódz. Mat. 24 (1993), 91-99.

22. J. Matkowski and J. Miś, On a characterization of Lipschitzian operators of substitution in the space $B V\langle a, b\rangle$, Math. Nachr. 117 (1984), 155-159.

23. N. Merentes, On a characterization of Lipschitzian operators of substitution in the space of bounded Riesz $\varphi$-variation, Ann. Univ. Sci. Budapest 34 (1991), 139-144. 
24. N. Merentes, Composing functions of bounded p-variation, Pure Math. Appl. 1 (1990), 39-45.

25. - On the composition operator in $A C[a, b]$, Collect. Math. 42 (1991), $121-127$.

26. N. Merentes and S. Rivas, On characterization of the Lipschitzian composition operator between spaces of functions of bounded p-variation, Czech. Math. J. 45 (1995), 627-637.

27. - Characterization of globally Lipschitzian composition operators between set-valued functions on $R V_{p}[a, b]$ and $R V_{q}[a, b]$, Publ. Math. Debrecen 47 (1995), 15-27.

28. - El operador de composición en espacios de funciones con algún tipo de variación acotada, Novena Escuela Venez. Mat., Mérida (Venezuela) 1996.

29. Kh.Sh. Mukhtarov, On the properties of the operator $F u=f(u(x))$ in the space $H_{\varphi}$, Sbor. Nauch. Rabot Mat. Kaf. Dagestan Univ. (1967), 145-150.

30. F. Riesz, Sur certains systèmes singuliers d'équations intégrales, Ann. Sci. Ecole Norm. Sup. Paris 28 (1911), 33-68.

31. A. Sieczko, Characterization of globally Lipschitzian Nemytskii operators in the Banach space $A C_{r-1}$, Math. Nachr. 141 (1989), 7-11.

32. E.P. Sobolevskij, The superposition operator in Hölder spaces, VINITI 376584, Voronezh 1984 (in Russian).

33. N. Wiener, The quadratic variation of a function and its Fourier coefficients, J. Math. Phys. MIT 3 (1924), 73-94.

Institute of Mathematics, University of Würzburg, Emil-Fischer-Str. 30, D-97074 Würzburg, Germany

Email address: jurgen@dmuw.de

School of Mathematics, Central University of Venezuela, Paseo Los Ilustres, Caracas 1020, Venezuela

Email address: nathaly.guanda@ciens.ucv.ve

Faculty of Mathematics, University of Voronezh, Universitetskaya pl. 1, R-394006 Voronezh, Russia

Email address: juli06@mail.ru 